\title{
Deformation fields near a steady fatigue crack with anisotropic plasticity
}

\author{
Yanfei Gao ${ }^{1,2, *}$ \\ ${ }^{1}$ Department of Materials Science and Engineering, University of Tennessee, Knoxville, TN 37996 \\ ${ }^{2}$ Materials Science and Technology Division, Oak Ridge National Laboratory, Oak Ridge, TN 37831
}

\begin{abstract}
In this work, from finite element simulations based on an irreversible, hysteretic cohesive interface model, a steady fatigue crack can be realized if the crack extension exceeds about twice the plastic zone size, and both the crack increment per loading cycle and the crack bridging zone size are smaller than the plastic zone size. The corresponding deformation fields develop a plastic wake behind the crack tip and a compressive residual stress field ahead of the crack tip. In addition, the Hill's plasticity model is used to study the role of plastic anisotropy on the retardation of fatigue crack growth and the elastic strain fields. It is found that for Mode-I cyclic loading, an enhanced yield stress in directions that are inclined from the crack plane will lead to slower crack growth rate, but this retardation is insignificant for typical degrees of plastic anisotropy. These results provide key inputs for future comparisons to neutron and synchrotron diffraction measurements that provide full-field lattice strain mapping near fracture and fatigue crack tips, especially in textured materials such as wrought or rolled $\mathrm{Mg}$ alloys.
\end{abstract}

Keywords: Fatigue; Cohesive interface simulations; Anisotropic plasticity

*Corresponding author: ygao7@utk.edu, or +1-865-974-2350. 
Essential concepts in fracture mechanics include the characterization of the relationship between the loading parameter (e.g., stress intensity factor, $K$ ) to the crack extension $\left(a-a_{0}\right.$ or $\Delta a)$, and the design of material microstructures to tune the process zones and thus the toughness $[1,2]$. The stress fields in an annular zone near the crack tip can be described by the product of the applied stress intensity factor, $K_{a p p l}$, the inverse square root singularity, $1 / \sqrt{2 \pi r}$ (with $r$ being the radial coordinate), and a set of characteristic fields that only depend on the polar angle, $\theta$. The lower bound of this $K$-annulus is set by the crack tip process zone, and the upper bound by the geometric boundaries. Examples of crack growth retardation or stress singularity shielding include plastic deformation, crack branching, fiber pull-out as a bridging mechanism, and phase transformation in the vicinity of the crack tip. The plastic process zone is "clean" since the rigorous mechanistic analysis can be conducted, while there exists a "messy" process zone at the crack tip and inside the plastic process zone where complicated damage mechanisms are still difficult to quantify. In the top-down approach by Hutchinson et al. [1,2], the "messy" process zone is represented by a cohesive interface model that relates the traction to the interface separation, so that the role of plastic deformation on fracture resistance can be quantitatively investigated. This approach naturally separates the scales dominated by $K$-field, "clean" plasticity-induced process zone, and "messy" damagerelated process zone, respectively. It also enables the development of a field projection approach from which the cohesive interface properties can be obtained from the far-field deformation measurements $[3,4]$.

The corresponding analysis of deformation fields near a fatigue crack tip is far more complicated because the process zone obviously traverses from the front to the hind of the crack tip. During the cyclic loading, the increase of the applied stress intensity factor to $K_{\max }$ leads to a plastic deformation region ahead of the fatigue crack tip, and the unloading from $K_{\max }$ and $K_{\min }$ leads to a reverse yield in a smaller zone, thus leading to a compressive residual stress in front of the crack $[5,6]$. Deformation behind the crack (i.e., the plastic wake) does not, however, permit such a simple analysis. In principle, one can adopt the top-down approach in $[1,2]$ to study the role played by the clean plastic process zone during fatigue loading, by 
assuming a set of cohesive interface constitutive laws for the messy damage process zone. The primary difficulty along this line lies on how to develop a phenomenological cohesive interface model that allows the simulation to numerically develop a steady fatigue crack. If successful, then the magnitude, distribution, and history of the deformation fields in the "intermediate" vicinity of the crack tip can be quantitatively related to the surrounding plasticity and the history of the applied stress intensity factors. On the other hand, the intrinsic fatigue mechanisms in the messy process zone involve cumulative damages and nucleation of flaws due to the large cyclic plastic deformation, which require quantitative analysis on the inter- and intra-granular scales [7]. Thus, the simulated deformation fields in the "immediate" vicinity of the fatigue crack tip will deviate from reality; if this deviation can be quantitatively measured, a mechanistic model of the cohesive interface behavior may become feasible. In the above, "intermediate" and "immediate" correspond to the clean and messy process zones, respectively.

The understanding of the fatigue crack growth behavior has been significantly enriched due to recent development of in situ, full-field, non-destructive measurements on microstructural lengths scales using neutron and synchrotron x-ray diffraction techniques [810]. Deformation fields on the grain scales are inhomogeneous. The shift of the diffraction peaks will give rise to the lattice strain, which relies on the intergranular interactions and the inhomogeneous deformation fields on the grain scale (or called Type-Il field). The peak broadening is related to the inhomogeneous deformation inside the grains, or called Type-III field. These measurements will help build the connection between the stress analysis from the top-down point of view and the failure mechanisms on the inter-and intra-granular scales from the bottom-up point of view. In synergy with the simulated deformation fields by the cohesive interface model, we can identify the regimes in which the simulations fail to reproduce the measured deformation field. And this comparison can be used to determine whether the phenomenological cohesive interface model can actually produce a steady fatigue crack with the correctly modeled plastic process zone, and also help develop a mechanism-based cohesive interface model. Simulation results in this paper will provide such benchmark inputs for future comparisons. 
Another important motivation of this work arises from textured alloys that are commonly used in engineering applications, e.g., metal forming and spring-back studies in textured steels, and fatigue and fracture studies in wrought or rolled $\mathrm{Mg}$ alloys. The plastic deformation is anisotropic, due to the initial texture, or polar nature of deformation twins, or pressure sensitivity that leads to yield asymmetry. Consequently, it is anticipated that the fatigue crack growth rate might vary with respect to the crack direction. It is thus of great technical importance to determine the deformation fields near a fatigue crack for these anisotropic plastic materials.

This work aims to develop a simulated steady fatigue crack, and to study the stress/strain distributions near the crack tip for Mises and anisotropic plastic solids. A cohesive interface is introduced along the crack path while the surrounding medium is elastic-plastic. The interface constitutive laws specify the relationship between the interface traction and the interface separation. Using such a top-down approach, a number of cohesive models have been proposed for the fatigue crack simulations [11-19]. Nguyen et al. [13] has demonstrated that the introduction of an irreversible cohesive law with unloading-reloading hysteresis is the simplest phenomenological way to model the accumulated crack tip damage and thus to realize a steady crack growth. In our cohesive interface model in [9] that is based on Nguyen et al. [13], we first specify the relationship between the normal traction $T_{n}$ and the normal separation $\Delta_{n}$ by

$$
\frac{T_{n}}{\sigma_{\max }}=\frac{\Delta_{n}}{\delta_{n}} \exp \left(1-\frac{\Delta_{n}}{\delta_{n}}\right)
$$

for the fully reversible behavior, and then introduce the unloading-reloading hysteresis by considering the unloading stiffness, $K^{-}$, and the reloading stiffness, $K^{+}$, separately,

$$
\begin{gathered}
\dot{T}_{n}= \begin{cases}K^{-} \dot{\Delta}_{n}, & \dot{\Delta}_{n}<0 \\
K^{+} \dot{\Delta}_{n}, & \dot{\Delta}_{n}>0\end{cases} \\
K^{-}=\frac{T_{n}^{\text {unload }}}{\Delta_{n}^{\text {unload }}},
\end{gathered}
$$




$$
\dot{K}^{+}= \begin{cases}\left(K^{+}-K^{-}\right) \dot{\Delta}_{n} / \delta_{a}, & \dot{\Delta}_{n}<0 \\ -K^{+} \dot{\Delta}_{n} / \delta_{f}, & \dot{\Delta}_{n}>0\end{cases}
$$

In the above equations, $\sigma_{\max }$ is the interface strength, $\delta_{n}$ is a characteristic length scale, $\delta_{a}$ and $\delta_{f}$ are length parameters that are used to tune the damage rate, and $T_{n}^{\text {unload }}$ and $\Delta_{n}^{\text {unload }}$ are the normal traction and separation when the unloading step starts. Since only Mode-I crack is considered in this work, the tangential response of the cohesive interface model is not specified.

The interface model in Eqs. (1)-(4) has been implemented as a user-defined element (UEL) subroutine in the commercial finite element software, ABAQUS $[8,9,20]$. A standard compact-tension (CT) specimen is used in our finite element simulations, with geometry specified in Fig. 1, which has a pre-notch in the middle and two loading pin holes for fatigue loading. Only half of the specimen is meshed due to symmetry with quadrilateral elements in the bulk and cohesive interface elements along the crack plane. Following the experiments on 316 stainless steel in [8], the specimen is assumed to be elastic and perfectly plastic with the Young's modulus $E=210 \mathrm{GPa}$, Poisson's ratio $v=0.3$, and yield stress $\sigma_{Y}=288 \mathrm{MPa}$. The interface parameters are $\sigma_{\max }=800 \mathrm{MPa}, \delta_{n}=1 \mu \mathrm{m}, \delta_{a} / \delta_{n}=0.4$, and $\delta_{f} / \delta_{n}=10$. From Eq. (4), it can be found that a large $\delta_{f} / \delta_{n}$ will prevent a rapid softening of $K^{+}$so as to slow down the crack growth rate, and a small $\delta_{a} / \delta_{n}$ will ensure that $K^{+}$approaches $K^{-}$rapidly during unloading. For example, we have found that the increase of $\delta_{f} / \delta_{n}$ from 4 to 20 leads to a decrease of the initial crack growth rate from $0.1 \mathrm{~mm} /$ cycle to $0.006 \mathrm{~mm} /$ cycle, for the CT specimen in Fig. 1 when subjected to a cyclic loading of $667 \sim 6667 \mathrm{~N}$. In order to realize a steady fatigue crack in our simulations, we have to ensure that the crack has grown out of the notch area of the CT specimen in Fig. 1, the crack extension is two or three times the plastic zone, and the crack increment per cycle and the crack bridging zone are smaller than the plastic zone. Also when simulating cracks using the cohesive interface model, numerical divergence may be encountered for small crack bridging zones that are often encountered in crack nucleation problem, which can be avoided by introducing a fictitious viscosity in Eq. (1) [21-23]. When all 
these conditions are met, the surrounding plastic field, not only ahead of the crack tip but also in the plastic wake, will be computed accurately in the intermediate vicinity of the crack tip and not depend on the particular choices of cohesive parameters - this is the advantage of the topdown approach that separates the "clean" plastic process zone and the "messy" damage process zone at the crack tip.

For clarity, Fig. 2 shows the schematic illustration of stress fields near a steady fatigue crack tip with Mises plasticity. Since the length is depicted by the plastic zone size, $r_{p}$, at $K_{\max }$, a dimensional analysis can be conducted to show that the stress distribution will depend on material parameters $\left(E / \sigma_{Y}, v\right.$, and hardening behavior), interface parameters $\left(\sigma_{\max } / \sigma_{Y}\right.$, $\delta_{a} / \delta_{n}$, and $\left.\delta_{f} / \delta_{n}\right)$, and the loading parameter $\left(K_{\min } / K_{\max }\right)$. As discussed in the preceding paragraph, $\delta_{a} / \delta_{n}$ and $\delta_{f} / \delta_{n}$ are chosen to ensure a steady fatigue crack with a crack bridging zone much smaller than the plastic zone. Therefore, the key parameters that dictate the stress distributions in Fig. 2 are $\sigma_{\max } / \sigma_{Y}$ and $K_{\min } / K_{\max }$. The latter is fixed to 0.1 as this ratio is typically used in fatigue tests. For a cohesive crack under monotonic loading, Tvergaard and Hutchinson [1] have shown that if $\sigma_{\max } / \sigma_{Y}$ is lower than about 3 for elastic perfectly plastic solid, no plastic deformation will occur near the crack tip. Results in Fig. 2 are for a ratio of $\sigma_{\max } / \sigma_{Y}=2.78$, which is sufficient to lead to the plastic process zone, and our tests with higher values $\sigma_{\max } / \sigma_{Y}$ found similar stress distributions but with slower crack growth rate. This issue will be addressed again when presenting results in Fig. 4. When comparing to the plastic zone near a stationary crack, results in the top plot in Fig. 2 clearly exhibit the plastic wake, whereas the Mises stress still remains high. The yield zone in front of the crack tip resembles a dumbbell, as opposed to the kidney shape in the stationary crack under a monotoic loading to $K_{\max }$. For $\sigma_{22}$ contours at $K_{\min }$, a compressive residual stress is found right ahead of the crack tip, in agreement with the Rice model in [5]. The Rice model, however, cannot predict the exact contour shapes or stresses in the plastic wake. Results in the bottom plot in Fig. 2 exhibit the extension of a compressive stress zone to the plastic wake, and a tensile stress at a distance of 
about $r_{p}$ in front of the crack tip. The magnitude of $\sigma_{22}$ field at $K_{\min }$ is about half of the yield stress, $\sigma_{Y}$.

Cohesive interface models for crack growth under monotonic loading by Hutchison et al. [1,2] and under cyclic loading presented in Fig. 2 suggest that one may manipulate the plastic anisotropy to retard the crack growth. For textured metals, there are certain directions in which the yield stresses are higher than other directions. For these materials, our numerical methodology can be used to determine the dependence of fatigue growth rate on the crack direction. We follow the definition of Hill's potential function in ABAQUS [20], given by

$$
f\left(\sigma_{i j}\right)=\sqrt{F\left(\sigma_{22}-\sigma_{33}\right)^{2}+G\left(\sigma_{33}-\sigma_{11}\right)^{2}+H\left(\sigma_{11}-\sigma_{22}\right)^{2}+2 L \sigma_{23}^{2}+2 M \sigma_{31}^{2}+2 N \sigma_{12}^{2}},
$$

where dimensionless parameters, $F, G, H, L, M$, and $N$ are defined as

$$
\begin{gathered}
F=\frac{1}{2}\left[\left(\frac{\sigma_{0}}{\bar{\sigma}_{22}}\right)^{2}+\left(\frac{\sigma_{0}}{\bar{\sigma}_{33}}\right)^{2}-\left(\frac{\sigma_{0}}{\bar{\sigma}_{11}}\right)^{2}\right]=\frac{1}{2}\left(\frac{1}{R_{22}^{2}}+\frac{1}{R_{33}^{2}}-\frac{1}{R_{11}^{2}}\right), \\
G=\frac{1}{2}\left[\left(\frac{\sigma_{0}}{\bar{\sigma}_{33}}\right)^{2}+\left(\frac{\sigma_{0}}{\bar{\sigma}_{11}}\right)^{2}-\left(\frac{\sigma_{0}}{\bar{\sigma}_{22}}\right)^{2}\right]=\frac{1}{2}\left(\frac{1}{R_{33}^{2}}+\frac{1}{R_{11}^{2}}-\frac{1}{R_{22}^{2}}\right), \\
H=\frac{1}{2}\left[\left(\frac{\sigma_{0}}{\bar{\sigma}_{11}}\right)^{2}+\left(\frac{\sigma_{0}}{\bar{\sigma}_{22}}\right)^{2}-\left(\frac{\sigma_{0}}{\bar{\sigma}_{33}}\right)^{2}\right]=\frac{1}{2}\left(\frac{1}{R_{11}^{2}}+\frac{1}{R_{22}^{2}}-\frac{1}{R_{33}^{2}}\right), \\
L=\frac{3}{2}\left(\frac{\tau_{0}}{\bar{\sigma}_{23}}\right)^{2}=\frac{3}{2 R_{23}^{2}}, M=\frac{3}{2}\left(\frac{\tau_{0}}{\bar{\sigma}_{13}}\right)^{2}=\frac{3}{2 R_{13}^{2}}, N=\frac{3}{2}\left(\frac{\tau_{0}}{\bar{\sigma}_{12}}\right)^{2}=\frac{3}{2 R_{12}^{2}} .
\end{gathered}
$$

In the above equation, $\bar{\sigma}_{i j}$ is the measured yield stress when $\sigma_{i j}$ is the only nonzero component of the applied stress, and $\sigma_{0}$ and $\tau_{0}=\sigma_{0} / \sqrt{3}$ are reference yield stress. The Hill model will degenerate to Mises plasticity if $\sigma_{0}=\sigma_{Y}$ and all the $R_{i j}$ ratios are unity. Although $R_{i j}$ are normally not much off unity, here we choose an extreme value of 2 for our numerical tests. In our plane strain problem, we only choose a certain direction, as indicated by the polar angle $\theta$, with the enhanced yield strength. That is, Figs. 3-5 simulate three cases: $R_{11}=2$ (i.e., 
$\theta=0^{\circ}$ ), or $R_{r r}=2$ at $\theta=45^{\circ}$, or $R_{22}=2$ (i.e., $\theta=90^{\circ}$ ). Results for $\theta=-45^{\circ}$ and $\theta=45^{\circ}$ will be symmetric about the $x_{1}$ axis. Our numerical simulations will adopt the same geometric setup as in Fig. 1, with the same interface parameters and also $\sigma_{0}$ being the Mises yield stress $\sigma_{Y}$ in Fig. 2.

The crack extension, $\Delta a$, versus the number of cycles, $N$, is plotted in Fig. 3 for the above three cases. All three curves are concave, indicating the increase of $d a / d N$ as the crack extends. Before $N \approx 450$, the case of $R_{r r}=2$ at $\theta=45^{\circ}$ has the slowest crack growth rate, but the difference from other cases is not large. We also note that all these cases will quickly lead to a fast crack growth upon $N>600$, mainly because the decrease of the ratio of $\sigma_{\max } / R_{r r} \sigma_{0}$ to 1.39 leads to a very weak interface. According to Hutchinson and Evans [2], such a ratio in a monotonically loaded crack with Mises plasticity will lead to cleavage crack without any plastic deformation surrounding the crack tip. In our case, we find that even at a distance several times than $r_{p}$, the normal stress may still exceed $\sigma_{\max }$ and thus lead to cumulative damage. Consequently, the crack growth rate will increase rapidly in later stage. No such behavior is observed in our simulations using a smaller $R$-ratio, such as $R_{r r}=1$. We should also point out that for textured alloys, the "messy" process zone may have different properties for different crack growth directions, which is rather not considered here. Nevertheless, even with the extreme value of $R_{r r}=2$ and with the same cohesive interface model, the crack growth behavior does not appear to be significantly affected by the plastic anisotropy.

It is anticipated that the resulting plastic zone will be different in these three cases because of the plastic anisotropy. As shown in Fig. 4, the middle case with $R_{11}=2$ appears to be almost the same as the Mises plasticity result in Fig. 2 . The top case with $R_{22}=2$ differs from Fig. 2 mainly in the plastic wake (which has a lower stress value). In this case, it is difficult for the normal stress to reach yield because of the enhanced yield strength in $x_{2}$ direction, so that the developed residual stress in the plastic wake is lower than that in the middle case. In 
contrast, the inclined case of $R_{r r}=2$ at $\theta=45^{\circ}$ rather resembles the stress distribution of a stationary case with very low stress value in the plastic wake.

Finite element simulations in the present work can provide valuable inputs for comparisons to diffraction measurements. It should be noted that diffraction measurements directly measured the lattice strains, which are elastic strains on a certain set of grains that satisfy the diffraction conditions. Thus in Fig. 5 , the contours of $\varepsilon_{22}^{\text {elastic }}$ are provided for these cases at $K_{\min }$. Among these results, the inclined case of $R_{r r}=2$ at $\theta=45^{\circ}$ has the most unusual distribution than the other two cases. The negative $\varepsilon_{22}^{\text {elastic }}$ near the notch root is a consequence of the geometric boundary, rather than a feature of a steady fatigue crack. In typical diffraction measurements such as in [8-10], the lattice strain mapping is restricted to the crack path, so that all these cases (Fig. 2 and Fig. 5) will give similar results. Provided with a fullfield mapping, one can identify the role of plastic anisotropy. We also note that these results are for an extreme value of $R$-ratio, while realistic $R$-ratios will certainly be bounded within the contrast between the top and bottom rows in Fig. 5. Also the quantitative study of the intermediate plastic process zone can be used to investigate the messy process zone, similar to the field projection technique $[3,4]$ that derives the cohesive properties from far fields. These important research lines will be left for future investigations.

In summary, an irreversible, hysteretic cohesive interface model is used in finite element simulations to develop a steady fatigue crack. This phenomenological model of the messy process zone allows us to study the clean process zone caused by the plastic deformation. This study extends the work by Hutchinson, Tvergaard and Evans [1,2] on the crack growth under monotonic loading. Our numerical simulations illustrate the development of plastic wake behind the crack and compressive residual stress ahead of the crack. When the surrounding plasticity is anisotropic, representative cases using Hill plasticity with extreme $R_{i j}$ ratios demonstrate only small variations in the crack growth rate and in the Mises stress contours at $K_{\max }$, but large differences in elastic strain contours are found at $K_{\min }$. These results provide 
benchmarks for full-field lattice strain mapping by advanced diffraction techniques such as neutron and synchrotron $\mathrm{x}$-ray for textured metals and alloys.

\section{Acknowledgements}

This work was sponsored by the U.S. Department of Energy, Office of Science, Basic Energy Sciences, Materials Sciences and Engineering Division. The author is grateful to Drs. W. $\mathrm{Wu}$ and $\mathrm{L}$. Zheng for fruitful discussions on fatigue cracks and neutron and synchrotron $\mathrm{x}$-ray diffraction measurements. 


\section{References}

[1] V. Tvergaard, J.W. Hutchinson, J. Mech. Phys. Solids 40 (1992) 1377.

[2] J.W. Hutchinson, A.G. Evans, Acta Mater. 48 (2000) 125.

[3] S. Hong, K.-S. Kim, J. Mech. Phys. Solids 51 (2003) 1267.

[4] H.B. Chew, J. Mech. Phys. Solids 61 (2013) 131.

[5] J.R. Rice, "The mechanics of crack tip deformation and extension by fatigue", in: Fatigue Crack Propagation, special technical publication 415, ASTM, Philadelphia, PA, (1967) 247-311.

[6] V. Tvergaard, Int. J. Fatigue 27 (2005) 1389.

[7] S. Suresh, Fatigue of Materials (1998), Cambridge University Press, UK.

[8] R.I. Barabash, Y.F. Gao, Y. Sun, S.Y. Lee, H. Choo, P.K. Liaw, D. Brown, G.E. Ice, Phil. Mag. Lett. 88 (2008) 553.

[9] L.L. Zheng, Y.F. Gao, S.Y. Lee, R.I. Barabash, J.H. Lee, P.K. Liaw, J. Mech. Phys. Solids 59 (2011) 2307.

[10] W. Wu, S.Y. Lee, A.M. Paradowska, Y.F. Gao, P.K. Liaw, Mater. Sci. Eng. A 556 (2012) 278.

[11] G.T. Camacho, M. Ortiz, Int. J. Solids Struct. 33 (1996) 2899.

[12] A. de-Andrés, J.L. Pérez, M. Ortiz, Int. J. Solids Struct. 36 (1999) 2231.

[13] O. Nguyen, E.A. Repetto, M. Ortiz, R.A. Radovitzky, Int. J. Fract. 110 (2001) 351.

[14] V.S. Deshpande, A. Needleman, E. van der Giessen, Acta Mater. 49 (2001) 3189.

[15] B. Yang, B. Mall, K. Ravi-Chandar, Int. J. Solids Struct. 38 (2001) 3927.

[16] K.L. Roe, T. Siegmund, Eng. Fract. Mech. 70 (2003) 209.

[17] Q.D. Yang, D.J. Shim, S.M. Spearing, Microelectron. Eng. 75 (2004) 85.

[18] S. Maiti, P.H. Geubelle, Eng. Fract. Mech. 72 (2005) 691. 
[19] S. Serebrinsky, M. Ortiz, Scripta Mater. 53 (2005) 1193.

[20] ABAQUS v6.3, user's manual, (2002) ABAQUS Inc., Rhode Island, USA.

[21] Y.F. Gao, A.F. Bower, Model. Simul. Mater. Sci. Eng. 12 (2004) 453.

[22] S.M. Xia, Y.F. Gao, A.F. Bower, L.C. Lev, Y.T. Cheng, Int. J. Solids Struct. 44 (2007) 3685.

[23] J.H. Lee, Y.F. Gao, K.E. Johanns, G.M. Pharr, Acta Mater. 60 (2012) 5448. 


\section{Figure Captions}

Figure 1 Cohesive interface simulations of a compact-tension (CT) specimen with geometric sizes taken from ASTM standards. The applied cyclic loading leads to alternating stress intensity factors, $K_{\max }$ and $K_{\min }$, with a ratio of $K_{\min } / K_{\max }=0.1$ in this work. Due to symmetry, only top half is meshed by quadrilateral elements and cohesive interface elements are added to the crack plane. The circle is the loading pin hole.

Figure 2 The stress contours plotted in the vicinity of a steady fatigue crack tip using a Mises plastic model. The steady fatigue crack is realized by ensuring the validity of a $K$ annulus at $K_{\max }$ and a crack extension that already exceeds twice the plastic zone size, $r_{p}$, at $K_{\max }$ (Top) Mises stress contours at $K_{\max }$ exhibit the formation of plastic wake behind the crack. (Bottom) Contours of normal stress $\sigma_{22}$ at $K_{\min }$ show a high compressive stress ahead of the crack tip, and a low compressive stress in the plastic wake. All contours are drawn to the same scale bar of $r_{p}$. Dashed boxes denote the crack bridging zone, i.e., our cohesive interface model of the messy process zone.

Figure 3 Crack extensions plotted against the number of cycles for Hill plastic solids. A Hill model with an anisotropic ratio of 2 in just one direction is adopted. This anisotropic direction is oriented in $\theta=0^{\circ}$ (i.e., $R_{11}=2$ ), $\theta=45^{\circ}$, and $\theta=90^{\circ}$ (i.e., $R_{22}=2$ ).

Figure 4 Mises stress contours at $K_{\max }$ for Hill plastic solids with three representative anisotropic directions. The scale bar is the plastic zone size for the Mises plastic solid under the same loading conditions at about the same crack extension (see Fig. 2). The deformation is magnified by a factor of 100 .

Figure 5 Contours of $\varepsilon_{22}^{\text {elastic }}$ at $K_{\text {min }}$ (unloaded right after $K_{\max }$ in Fig. 4) for Hill plastic solids with three representative anisotropic directions. The scale bar is the plastic zone size for the Mises plastic solid under the same loading conditions at about the same crack extension (Fig. 2). The deformation is magnified by a factor of 100 . 


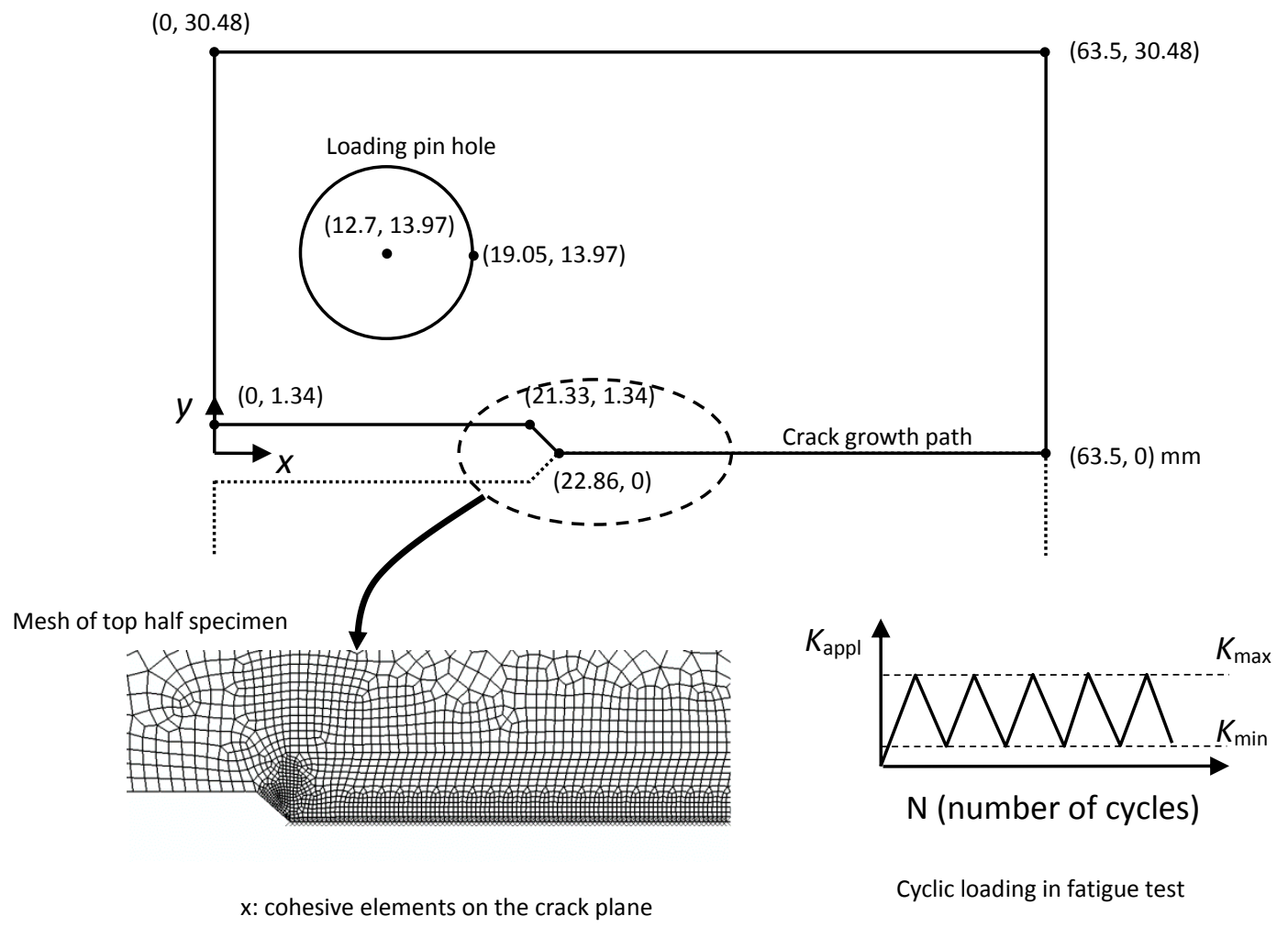


Figure 1

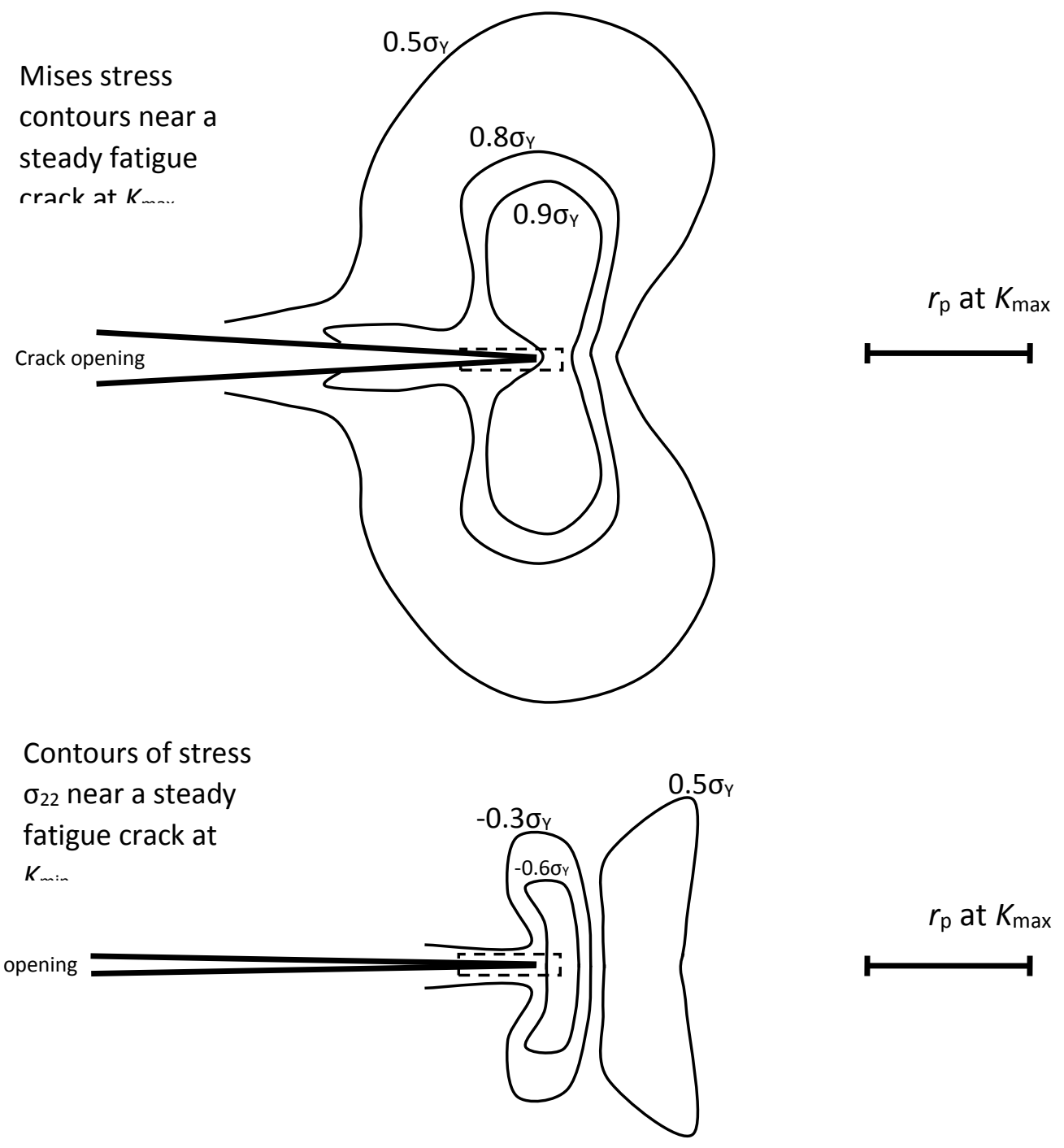


Figure 2

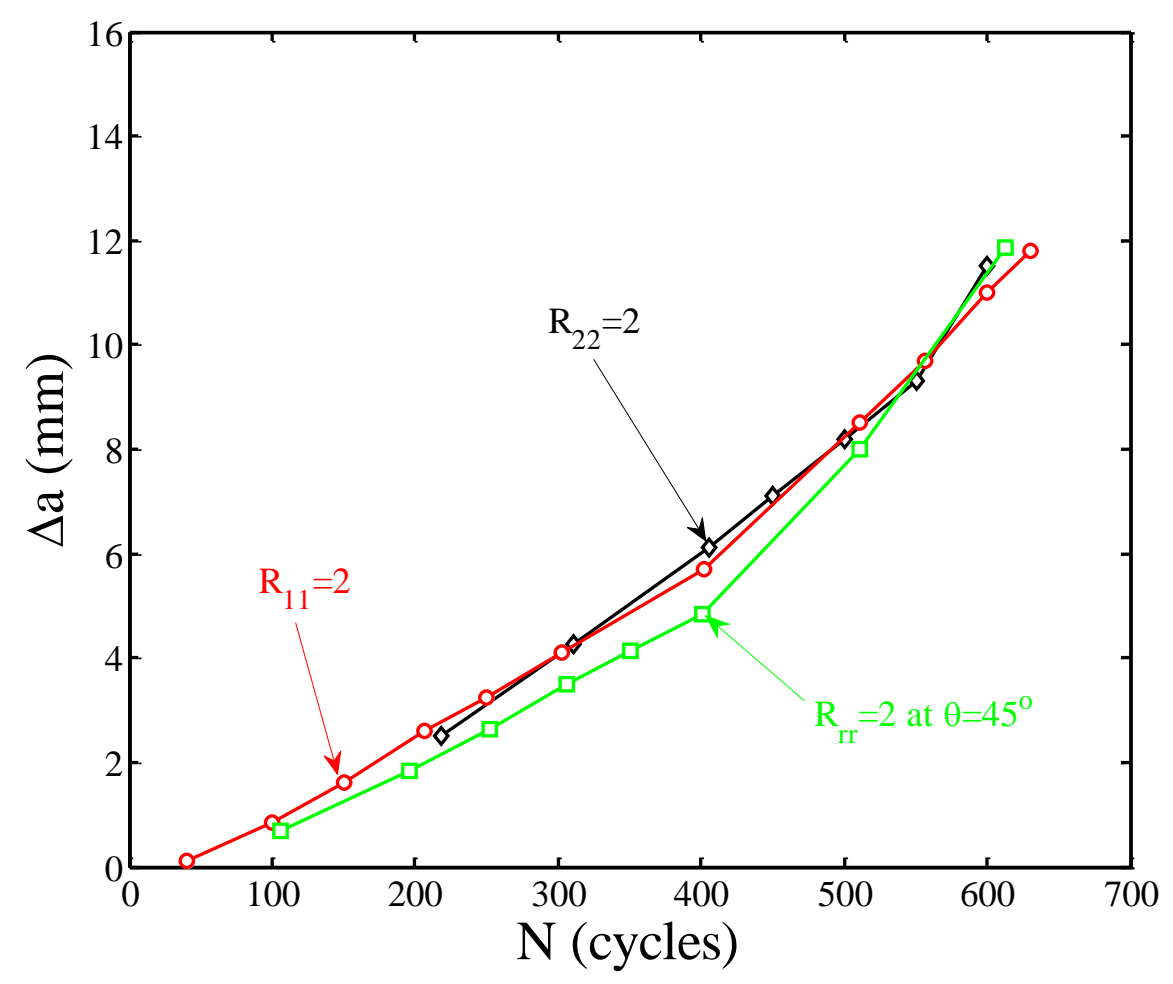


Figure 3 


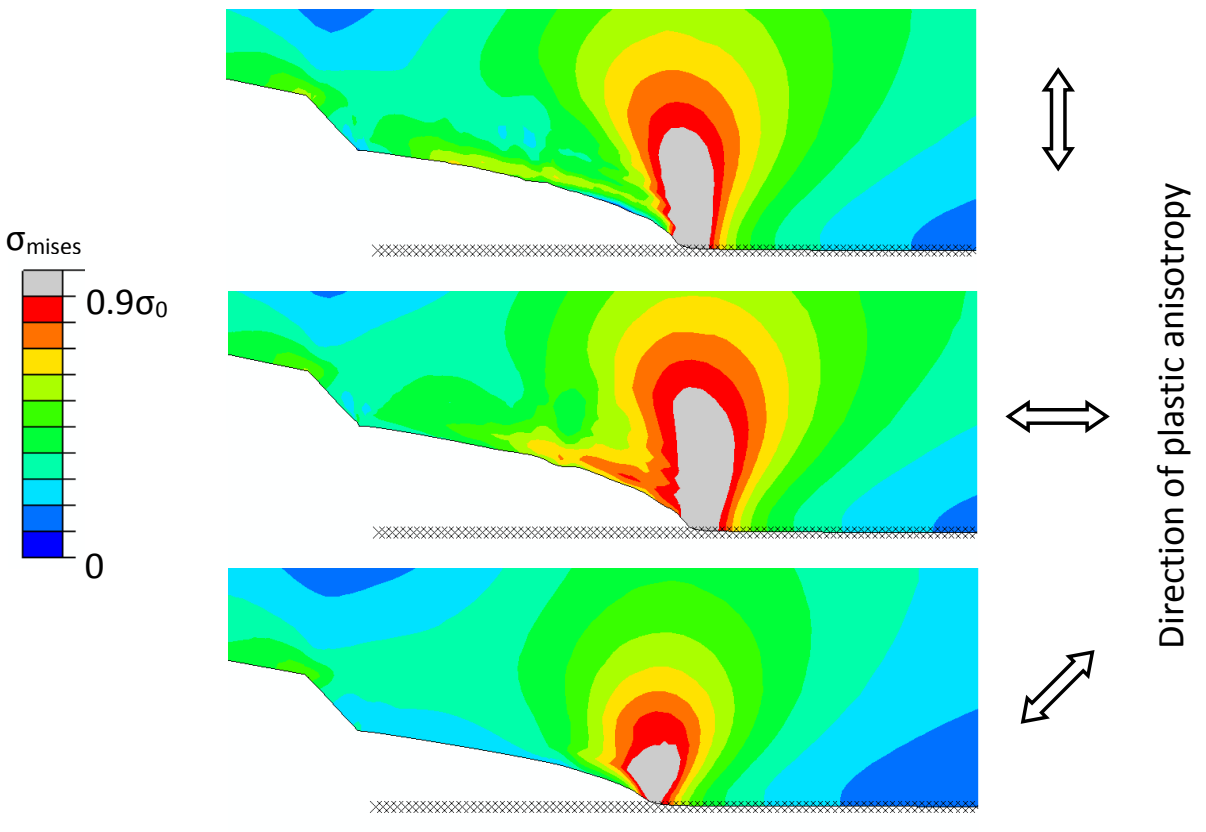

$r_{\mathrm{p}}$ of Mises solid at 
Figure 4
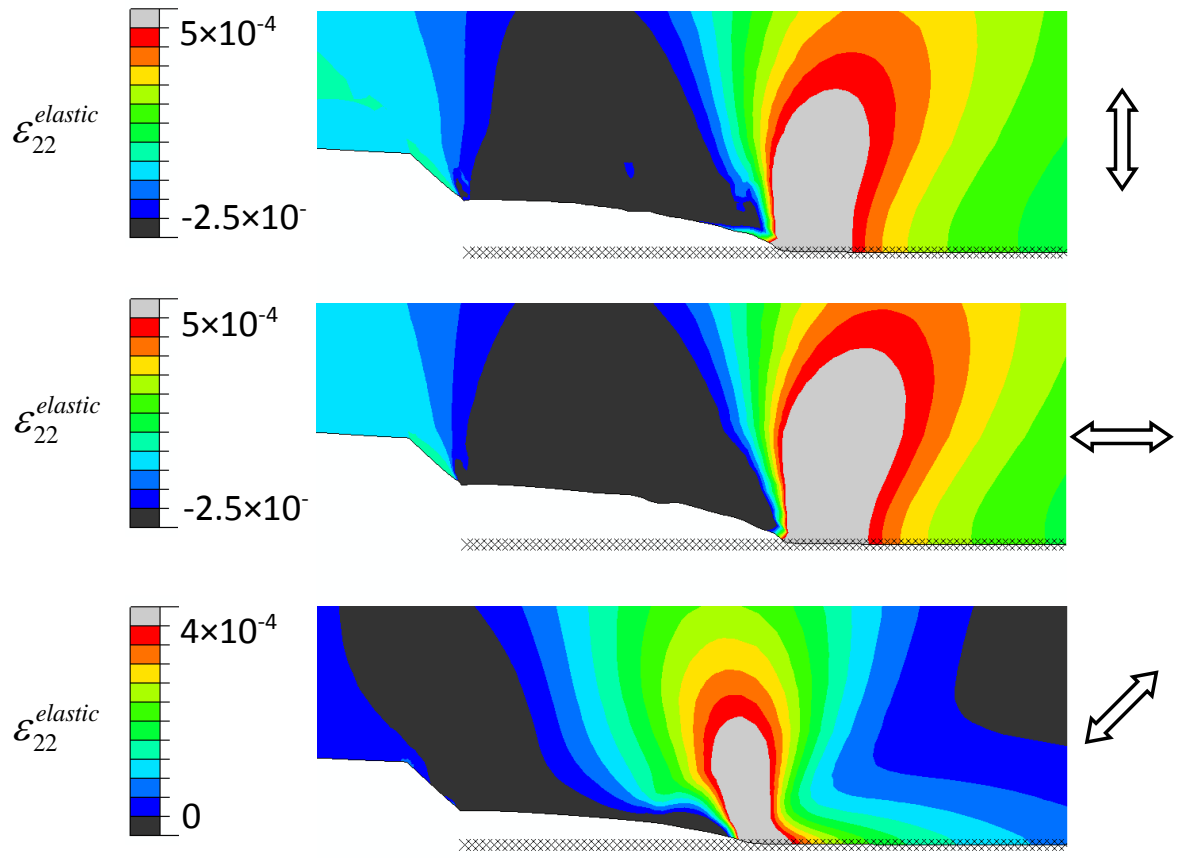

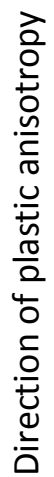

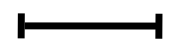

$r_{p}$ of Mises solid at 
Figure 5 